Published in final edited form as:

JAMA Intern Med. 2016 March ; 176(3): 329-337. doi:10.1001/jamainternmed.2015.8033.

\title{
A Mind-Body Program for Older Adults With Chronic Low Back Pain:
}

\section{A Randomized Clinical Trial}

Natalia E. Morone, MD, MS,

Division of General Internal Medicine, Center for Research on Health Care, University of Pittsburgh School of Medicine, Pittsburgh, Pennsylvania; Clinical and Translational Sciences Institute, University of Pittsburgh School of Medicine, Pittsburgh, Pennsylvania; Geriatric Research Education and Clinical Center, Veterans Affairs Pittsburgh Healthcare System, Pittsburgh, Pennsylvania

Carol M. Greco, PhD, Department of Psychiatry, Center for Integrative Medicine at University of Pittsburgh Medical Center (UPMC), University of Pittsburgh School of Medicine, Pittsburgh, Pennsylvania

Charity G. Moore, PhD, Dickson Advanced Analytics, Carolinas HealthCare System, Charlotte, North Carolina

\section{Bruce L. Rollman, MD, MPH,} Division of General Internal Medicine, Center for Research on Health Care, University of Pittsburgh School of Medicine, Pittsburgh, Pennsylvania; Clinical and Translational Sciences Institute, University of Pittsburgh School of Medicine, Pittsburgh, Pennsylvania

\section{Bridget Lane, MA,}

Division of General Internal Medicine, Center for Research on Health Care, University of Pittsburgh School of Medicine, Pittsburgh, Pennsylvania

Corresponding Author: Natalia E. Morone, MD, MS, Division of General Internal Medicine, Center for Research on Health Care, University of Pittsburgh School of Medicine, 230 McKee Pl, Ste 600, Pittsburgh, PA 15213 (moronene@upmc.edu).

Author Contributions: Dr Morone had full access to all the data in the study and takes responsibility forthe integrity of the data and the accuracy of the data analysis.

Study concept and design: Morone, Greco, Moore, Weiner.

Acquisition, analysis, or interpretation of data: Morone, Moore, Rollman, Lane, Morrow, Glynn, Weiner.

Drafting of the manuscript: Morone, Moore.

Critical revision of the manuscript for important intellectual content: All authors.

Statistical analysis: Moore.

Obtained funding: Morone.

Administrative, technical, or material support: Morone, Greco, Rollman, Lane, Glynn.

Study supervision: Morone, Greco, Weiner.

Additional Contributions: The following members of the Aging Successfully with Pain study team contributed to this study: Laura Kinzel, MS, RD, and Daniel Schenck, DC, participated as program instructors; Lori Thiry, BS, Deanna Matthew, BS, and Vincent

Losasso, MS, participated as research assistants. Their salaries were paid by the grant.

Conflict of Interest Disclosures: None reported.

Role of the Funder/Sponsor: The funding source had no role in the design and conduct of the study; collection, management, analysis, and interpretation of the data; preparation, review, or approval of the manuscript; and decision to submit the manuscript for publication.

Disclaimer: The content is solely the responsibility of the authors and does not necessarily represent the official views of the National Institute on Aging, the Department of Veterans Affairs, or the US government.

TRIAL REGISTRATION clinicaltrials.gov Identifier: NCT01405716 


\author{
Lisa A. Morrow, PhD, \\ Department of Psychiatry, University of Pittsburgh School of Medicine, Pittsburgh, Pennsylvania
}

Nancy W. Glynn, PhD, and

Department of Epidemiology, Graduate School of Public Health, University of Pittsburgh,

Pittsburgh, Pennsylvania

\title{
Debra K. Weiner, MD
}

Clinical and Translational Sciences Institute, University of Pittsburgh School of Medicine, Pittsburgh, Pennsylvania; Geriatric Research Education and Clinical Center, Veterans Affairs

Pittsburgh Healthcare System, Pittsburgh, Pennsylvania; Department of Psychiatry, University of Pittsburgh School of Medicine, Pittsburgh, Pennsylvania; Department of Anesthesiology, University of Pittsburgh School of Medicine, Pittsburgh, Pennsylvania; Division of Geriatric Medicine, Department of Medicine, University of Pittsburgh School of Medicine, Pittsburgh, Pennsylvania

\section{Abstract}

IMPORTANCE-Treatment of chronic low back pain (LBP) in older adults is limited by the adverse effects of analgesics. Effective nonpharmacologic treatment options are needed.

OBJECTIVE-To determine the effectiveness of a mind-body program at increasing function and reducing pain in older adults with chronic LBP.

DESIGN, SETTING, AND PARTICIPANTS-This single-blind, randomized clinical trial compared a mind-body program $(n=140)$ with a health education program $(n=142)$.

Community-dwelling older adults residing within the Pittsburgh metropolitan area were recruited from February 14, 2011, to June 30, 2014, with 6-month follow-up completed by April 9, 2015. Eligible participants were 65 years or older with functional limitations owing to their chronic LBP ( $>11$ points on the Roland and Morris Disability Questionnaire) and chronic pain (duration $>3$ months) of moderate intensity. Data were analyzed from March 1 to July 1, 2015.

INTERVENTIONS-The intervention and control groups received an 8-week group program followed by 6 monthly sessions. The intervention was modeled on the Mindfulness-Based Stress Reduction program; the control program, on the "10 Keys" to Healthy Aging.

MAIN OUTCOMES AND MEASURES-Follow-up occurred at program completion and 6 months later. The score on the Roland and Morris Disability Questionnaire was the primary outcome and measured functional limitations owing to LBP. Pain (current, mean, and most severe in the past week) was measured with the Numeric Pain Rating Scale. Secondary outcomes included quality of life, pain self-efficacy, and mindfulness. Intent-to-treat analyses were conducted.

RESULTS-Of 1160 persons who underwent screening, 282 participants enrolled in the trial (95 men [33.7\%] and 187 women [66.3\%]; mean [SD] age, 74.5 [6.6] years). The baseline mean (SD) Roland and Morris Disability Questionnaire scores for the intervention and control groups were 15.6 (3.0) and 15.4 (3.0), respectively. Compared with the control group, intervention participants improved an additional -1.1 (mean, 12.1 vs 13.1 ) points at 8 weeks and -0.04 (mean, 12.2 vs 12.6) points at 6 months (effect sizes, -0.23 and -0.08 , respectively) on the Roland and Morris 
Disability Questionnaire. By 6 months, the intervention participants improved on the Numeric Pain Rating Scale current and most severe pain measures an additional -1.8 points (95\% CI, -3.1 to -0.05 points; effect size, -0.33 ) and -1.0 points ( $95 \%$ CI, -2.1 to 0.2 points; effect size, -0.19 ), respectively. The changes in Numeric Pain Rating Scale mean pain measure after the intervention were not significant ( -0.1 [95\% CI, -1.1 to 1.0 ] at 8 weeks and -1.1 [95\% CI, -2.2 to -0.01$]$ at 6 months; effect size, -0.01 and -0.22 , respectively).

CONCLUSIONS AND RELEVANCE-A mind-body program for chronic LBP improved shortterm function and long-term current and most severe pain. The functional improvement was not sustained, suggesting that future development of the intervention could focus on durability.

Chronic pain affects approximately 100 million adults in the United States. ${ }^{1}$ Among adults 65 years and older, the prevalence of pain was found to be $52.9 \%$ in the National Health and Aging Trends Study. ${ }^{2}$ The back was the most common site and identified in $30.3 \%$ of the sample. Those adults with pain exhibited decreased physical function compared with those without pain. Treatment is often unsatisfactory in older adults, in part owing to the adverse effects of non-steroidal anti-inflammatory drugs or opioids. Fewer treatment options may lead to the prescription of invasive interventions that may be of limited benefit. ${ }^{3}$ Additional nonpharma-cologic treatment options are needed.

According to the most recent National Health Interview Survey, 18 million Americans tried meditation in 2012. ${ }^{4}$ This uptake of meditation is associated with a growing evidence base on its health benefits. In particular, a recent meta-analysis found mindfulness meditation has a moderate effect on decreasing pain, anxiety, and depression. ${ }^{5}$ The effect on pain found in this study was primarily owing to a reduction in visceral pain, whereas the effect on musculoskeletal pain was not clinically significant. However, the latter finding was based on only 2 studies with small sample sizes. ${ }^{6,7}$ Therefore, further evidence of the effects of mindfulness meditation on musculoskeletal pain conditions is needed.

A pilot study in older adults with chronic low back pain (LBP) demonstrated the feasibility of an 8-week mind-body program that taught commonly used methods of mindfulness meditation. In addition, initial evidence of a treatment effect for decreasing pain and increasing function and quality of life has been noted. ${ }^{6,8}$ We present the next phase of this research, which was to determine the effectiveness of mindfulness meditation in an adequately powered clinical trial. The primary aim of the study was to determine the effectiveness of the mind-body program at increasing function and reducing pain in adults 65 years or older with chronic LBP. We hypothesized that mindfulness meditation would be associated with a clinically meaningful improvement in physical function after the 8-week mind-body program compared with a health education control program. We also hypothesized that compared with the control group, intervention participants would experience decreased pain and increased psychological function immediately after the 8week mind-body program, and that 6 months after program completion, the magnitude of improvement in physical function, pain, and psychological function would be maintained. 


\section{Methods}

\section{Overview}

This experimental study was designed as a randomized, education-controlled clinical trial of a mind-body program for older adults with chronic LBP. Details of the study procedure have been published previously. ${ }^{9}$ We randomized 282 independent, community-dwelling adults 65 years or older who were recruited from metropolitan Pittsburgh, Pennsylvania. Participants in the mind-body (intervention) group received the group intervention of 8 weekly 90-minute mindfulness meditation sessions modeled on the Mindfulness-Based Stress Reduction program. ${ }^{10}$ Controls received an 8 -week group health education program based on the "10 Keys" to Healthy Aging. ${ }^{11}$ After completion of the 8-week program, participants in the intervention and control programs were asked to return for 6 monthly booster sessions. Measures were obtained at baseline, after the 8-week program, and 6 months after program completion. Recruitment occurred from February 14, 2011, to June 30, 2014. The final 6-month assessment was completed April 9, 2015. The study protocol was approved by the institutional review board of the University of Pittsburgh. All participants provided written informed consent.

\section{Inclusion and Exclusion Criteria}

Participants were included if they were 65 years or older, spoke English, had intact cognition (Mini-Mental State Examination score, 224$),{ }^{12}$ had functional limitations owing to their chronic LBP (defined as a score of $\geq 11$ on the Roland and Morris Disability Questionnaire [RMDQ]; range, 0-24, with higher scores indicating increased limitations), ${ }^{13}$ and had selfreported moderate chronic pain levels on a verbal descriptor scale (Pain Thermometer; measured on a visual scale as pain as bad as it could be, extreme, severe, moderate, mild, or no pain) occurring daily or almost every day for at least the previous 3 months. ${ }^{13}$ Participants were excluded if they had participated in a previous mindfulness meditation program, had serious underlying illness (such as malignant neoplasms, infection, unexplained fever, weight loss, or recent trauma) causing their pain, were nonambulatory, had severe impaired mobility, had visual or hearing impairment that interfered with assessments, had pain in other parts of the body more severe than their chronic LBP or acute back pain, had an acute or a terminal illness, or had moderate to severe depressive symptoms (Geriatric Depression Scale score, 21 ; range, 0-30). ${ }^{14}$

\section{Assessments and Outcome Measures}

The RMDQ was the primary outcome measure. It contains 24 questions specifically related to functional limitations as a result of LBP. A clinically meaningful change on the RMDQ ranges from a 2.5- to a 5.0-point improvement (reduction) from baseline. ${ }^{15}$ Pain (present, average, and most severe during the past week) was measured by self-report with the Numeric Pain Rating Scale (NRS; range, 0-20, with higher scores indicating worse pain). ${ }^{16}$ Because pain is a complex phenomenon that affects quality of life, mood, and psychological function, we chose a variety of established instruments to measure these different domains. Quality of life was measured with the RAND-36 Health Status Inventory (Global Health Composite [range, 9-67, with higher scores indicating better global health]; and Physical Health Composite [range, 20-65, with higher scores indicating better physical health]). ${ }^{17}$ 
Given the strong association between chronic pain and depression, ${ }^{18}$ we used the Geriatric Depression Scale. Self-efficacy has been shown to predict task performance. ${ }^{19}$ We measured this construct with the well-validated Chronic Pain Self-Efficacy Scale (range, 0\%-100\%, with higher scores indicating improved self-efficacy). ${ }^{20}$ Pain catastrophizing was measured with the Catastrophizing Scale of the Coping Strategies Questionnaire (range, 0-6, with higher scores indicating greater catastrophizing). ${ }^{21}$ We assessed self-reported mindfulness with the Mindful Attention Awareness Scale (MAAS; range, 1-6, with higher scores indicating greater mindfulness). ${ }^{22}$ Data on comorbidity were reported with the Cumulative Illness Rating Scale (range, 0-13, with higher scores indicating more comorbid conditions).

${ }^{23}$ Expectancy and credibility were assessed at baseline. ${ }^{24}$ Assessment of primary and secondary outcome measures occurred immediately ( 8 weeks) and 6 months after program completion.

To examine the process of change, brief (15-minute) monthly telephone evaluations of major domains (function, pain, mindfulness, and health system encounters) were conducted. Health system encounters were determined by monthly telephone calls that included questions regarding the use of health care services. All encounters that involved hospitalization or emergency department visits were reviewed by the principal investigator (N.E.M.) and by the data safety monitoring board (which included a physician) to determine whether the reasons constituted an adverse event related to the intervention or control programs.

\section{Randomization and Blinding}

After initial telephone screening, in-person written informed consent was obtained. If participants were still eligible after screening for physical function, cognition, and depression, a physical examination and baseline measures were completed. All eligible research participants were randomized into the intervention or the control group after baseline measures were obtained. The initial randomization was generated by the study statistician (C.G.M.) using a permuted block design with a block size of 4 in a 1:1 ratio using SAS PROC PLAN software (SAS Institute, Inc). The list was uploaded into the Webbased data collection system and concealed such that each allocation was only provided after a participant was deemed eligible. After 155 participants had been randomized, a significant imbalance between groups by sex was noted. With approval from the data safety monitoring board, we changed the fixed randomization to a baseline adaptive randomization to balance future allocations by sex and corrected the imbalance. ${ }^{25}$ The Web-based data collection system was modified to randomize in real time using the minimization method rather than the fixed list. Only after baseline measures were completed was the allocation available for access by the project coordinator (B.L.) who then communicated the assignment to the participant in person or by telephone. All outcome assessments were conducted by staff members blinded to intervention assignment.

\section{Intervention}

The intervention was modeled on the 8-week Mindfulness-Based Stress Reduction program. Four methods of mindful-ness meditation were taught. These techniques take regular activities such as sitting, walking, and lying down and transform them into a meditation 
through directed breathing and mindful awareness of thoughts and sensations. The methods used included the body scan, sitting practice, walking meditation, and mindful stretching. To encourage proficiency with the meditation method after completion of the intervention, monthly 60-minute booster sessions were held. Each session included time for a mindfulness meditation and time for discussion of the themes brought up during the 8-week program.

\section{Control Program}

The comparison group controlled for time, group size, attention, homework, and facilitator time. We based the 8-week health education program on a successful aging curriculum known as the 10 Keys to Healthy Aging. ${ }^{11}$ Pain information is not a component of the 10 Keys to Healthy Aging program, and we did not add this information to avoid contamination of the control group. The 10 Keys to Healthy Aging teaches an interactive, dynamic program to older adults on key health topics relevant to healthy aging such as hypertension management. The same chair stretches taught in the intervention were taught in the control program. To ensure that the control group received an equal amount of attention and social support, they also received monthly booster classes, which met monthly for 1 hour. The classes were based on the topics presented in the 10 Keys to Healthy Aging and presented by local experts in the field.

\section{Sample Size}

With a sample size of 300 , our study was powered (80\%-90\%) to detect a 2.5-point difference on the RMDQ between the 2 study arms at 8 weeks, assuming an SD of 5.9, intraclass correlation of 0.01 to 0.02 , and $20 \%$ attrition. ${ }^{9}$ Our attrition rate at 8 weeks was much lower than expected (4.3\%), therefore we stopped recruitment after 282 individuals had been randomized. We conservatively anticipated an approximate loss to or unavailability for follow-up of 28 participants (10\%) at 8 weeks, which would have provided data on at least 254 participants and maintained our original power.

\section{Statistical Analysis}

Data were analyzed from March 1 to July 1, 2015. The statistical analysis plan has been described previously. ${ }^{9}$ A brief summary of the analyses used for this presentation of the primary results follows. All analyses were conducted using intention to treat and with 2sided tests of $\mathrm{a}=.05$. We compared baseline characteristics of the participants in the intervention and control programs using 2-sample $t$ tests (or Wilcoxon rank sum tests) for continuous variables and $\chi^{2}$ tests (or Fisher exact tests) for categorical variables. As originally proposed, we used linear mixed models to compare the RMDQ scores at the baseline, 8-week, and 6-month assessments in the outcome vector. We controlled for repeated measures using autoregressive covariance structure and controlled for correlation among individuals within the same class cohort (intraclass correlation) using a random class effect. The fixed effects were group (intervention or control), time, the interaction of group by time, and sex (owing to randomization stratification). We used contrasts to estimate and test the adjusted difference between the intervention and control groups at each point. Secondary outcomes that were continuous were analyzed using the same approach. To compare the proportion of participants with a clinically meaningful response on the RMDQ, NRS, and a global impression of change, the outcomes were dichotomized and tested using 
$\chi^{2}$ analyses at each point. An interim analysis for the primary outcome was not conducted throughout the course of the trial. All analyses were conducted in SAS statistical software (version 9.3; SAS Institute, Inc).

\section{Results}

Of the 1160 persons who underwent screening, 282 met all eligibility criteria and provided signed informed consent to participate (mean [SD] age, 74.5 [6.6] years). Of these, we randomized 140 to the intervention program and 142 to the control program (Figure 1). The intervention was not delivered to 4 participants because 2 were no longer interested in participating in the study, 1 developed a serious illness, and 1 had unexpected family obligations. Five participants did not receive the control program because 2 were no longer interested, 1 developed unexpected illness, 1 had an unexpected family illness, and 1 was not satisfied with the randomization assignment. However, 2 of these participants from the intervention group and 3 from the control group agreed to complete follow-up assessments. At program completion (8 weeks), 132 participants in the intervention group (94.3\%) and 138 in the control group (97.2\%) completed assessments. Six months after program completion, 118 in the intervention group (84.3\%) and 135 in the control group (95.1\%) underwent assessment. Both groups were similar on all baseline variables (Table 1).

Participants attended a mean of 6.6 sessions for each group (range, $0-8$ sessions). Participants attended a mean of 2.4 booster sessions in the intervention group and 2.5 in the control group (range, $0-6$ booster sessions). No adverse events related to the intervention or control group were noted.

\section{Outcomes}

The baseline mean (SD) RMDQ scores for the intervention and control groups were 15.6 (3.0) and 15.4 (3.0), respectively. Compared with the control group, participants in the intervention group improved an additional -1.1 points on the RMDQ at 8 weeks and -0.4 points at 6 months (overall group $\times$ time interaction, $P=.01$ ) (Table 2). The intervention group had a mean change of -3.5 points on the RMDQ at 8 weeks and -3.4 points at 6 months, whereas the control group a mean change of -2.3 points at 8 weeks and -2.8 points at 6 months. The corresponding effect sizes between groups at 8 weeks and 6 months were -0.23 and -0.08 , respectively. The effect of the intervention on function was not significantly modified by age, race, educational level, or sex. Per protocol missing data methods were applied with no effect on the inference for the RMDQ. Participants in the intervention group significantly improved over time on the current and most severe NRS pain measures for the past week compared with those in the control group, with an additional -1.8- and -1.0-point improvement by 6 months, respectively. This improvement corresponded to effect sizes between groups at 8 weeks and 6 months of -0.33 and -0.19 , respectively. Differences on the average NRS pain measure between groups did not reach statistical significance $(P=.08)$.

Seventy-five of 132 intervention participants (56.8\%) vs 62 of 138 control participants $(44.9 \%)$ had at least a 2.5 -point clinically significant improvement at 8 weeks, but this difference did not reach statistical significance $(P=.051)$. At 6 months, 58 of 117 
intervention participants (49.2\%) and 66 of 135 control participants (48.9\%) hada 2.5-point improvement $(P=.97)$ (Table 3). Seven participants in the intervention group (5.3\%) and 6 participants in the control group (4.3\%) had at least a 2.5-point worsening on the RMDQ at 8 weeks. Six participants in the intervention group (5.1\%) and 6 participants in the control group (4.4\%) had at least a 2.5 point worsening on the RMDQ at 6 months.

In addition to the mean change over time in the pain scores, we evaluated the proportion of participants with a clinically meaningful $30 \%$ improvement. We found that immediately after program completion, more participants in the intervention group compared with the control group achieved a 30\% improvement on the current (54 of 132 [40.9\%] vs 34 of 138 [24.6\%]; $P=.004$ ) and most severe (48 of 132 [36.4\%] vs 30 of 138 [21.7\%]; $P=.008$ ) NRS pain measures for the past week. Similar differences were found at 6 months for the current (52 of 117 [44.4\%] vs 34 of 135 [25.2\%]; $P=.001$ ) and most severe (42 of 117 [35.9\%] vs 30 of 135 [22.2\%]; $P=.02$ ) (Table 3) NRS pain measures. We also evaluated a more stringent 50\% improvement on the average (21 of 132 [15.9\%] vs 14 of 138 [10.1\%]; $P=$. 16), current (43 of 132 [32.6\%] vs 22 of 138 [15.9\%]; $P=.001$ ), and most severe (21 of 132 [15.9\%] vs 12 of 138 [8.7\%]; $P=.07$ ) NRS pain measures and found a significant difference between groups for current pain only at program completion. The intervention group had a higher proportion achieving a 50\% improvement at 6 months for the average (29 of 117 [24.8\%] vs 18 of 135 [13.3\%]; $P=.02$ ) and current (41 of 117 [35.0\%] vs 28 of 135 [20.7\%]; $P=.01$ ) but not most severe (25 of 117 [21.4\%] vs 17 of 135 [12.6\%]; $P=.06$ ) NRS pain measures.

We assessed the global impression of change scores at program completion (Figure 2). The intervention participants reported more improvement in their back pain symptoms compared with the control participants $(P<.001)$. At 8 weeks, 106 of 132 participants $(80.3 \%)$ in the intervention program had at least minimal improvement, compared with 51 of 138 (37.0\%) in the control program. In the control program, 84 participants (61.0\%) reported no change and $3(2.2 \%)$ reported worsened symptoms. Notably, 54 participants in the intervention group $(40.9 \%)$ were much or very much improved compared with $9(6.5 \%)$ in the control group. This difference persisted at 6 months in the intervention group, with a greater number of participants reporting worsening symptoms in the control group (eFigure 1 in the Supplement). Credibility and expectancy of the programs to reduce back pain was obtained at baseline, with no difference between groups (eFigure 2 in the Supplement).

Secondary outcome measures are also presented in Table 2. The Global Health Composite and Physical Health Composite scores of the RAND-36 Health Status Inventory by 6 months improved an additional 0.2 ( -1.9 to 2.4 ) points and -0.01 ( -1.9 to 1.8 ) points, respectively, for the intervention group. The improvement in pain self-efficacy subscales at 8 weeks was not sustained at the 6-month assessment. Pain catastrophizing effect sizes were -0.19 and 0.05 at 8 weeks and 6 months, respectively. The intervention showed no improvement in self-reported mindfulness or depressive symptoms. 


\section{Discussion}

We found that the intervention group significantly improved in short-term function at 8 weeks, but the functional improvement was not sustained. The intervention group also showed significantly reduced current and most severe pain for the past week during the course of the 6-month follow-up. Although some secondary outcomes such as pain selfefficacy showed improvement in the intervention compared with the control group immediately after program completion, these differences were not sustained. Self-reported mindfulness did not change between groups, and changes in quality of life and pain catastrophizing did not reach clinical significance.

Preserving physical function with aging is critical to maintaining independence, because loss of independence is arguably one of the most feared consequences of aging. Learning mindfulness resulted in a clinically meaningful mean RMDQ improvement of -3.4 points, but by 6 months, the comparison group had also improved by a mean of -2.8 points. This improvement corresponded to $49.2 \%$ and $48.9 \%$ of participants in the respective groups with a clinically meaningful improvement in function. Physical activity was not a part of either program, although both groups learned a 10-minute chair stretching exercise during class 3 .

The mindfulness group also had a statistically and clinically significant $30 \%$ improvement in current and most severe (in the past week) pain intensity compared with the control group. These findings held up for current pain, when a 50\% improvement was determined. This amount of improvement in pain is consistent with a patient-centered view of successful pain relief. ${ }^{26}$ However, many therapies do not achieve this level of pain reduction. A 2006 Cochrane Review of acetaminophen vs placebo for hip or knee osteoarthritis pain ${ }^{27}$ found that patients experienced a statistically significant reduction with multiple methods used to evaluate pain compared with placebo, with the relative change from baseline of 5\%. A 2005 meta-analysis of exercise therapy for nonspecific $\mathrm{LBP}^{28}$ found that the therapy was effective relative to comparisons and that the pooled mean improvement for patients was 13.3 of 100 points for pain and 6.9 of 100 points for function.

Participants also perceived improvement in pain as a result of the intervention. One hundred six participants (80.3\%) described improvement in their back pain symptoms immediately after the 8-week intervention compared with 51 (37.0\%) in the control group. At 6 months, $89(76.1 \%)$ stated at least minimal improvement compared with $57(42.2 \%)$ in the control group.

The 8-week mind-body program resulted in an increase in pain self-efficacy immediately after program completion. A recent meta-analysis found that higher levels of self-efficacy were associated with decreased impairment, affective distress, and pain severity among patients with chronic pain. ${ }^{29}$ Improving self-efficacy is an important component of teaching patients how to cope with pain. Our intervention program successfully accomplished this coping, but self-efficacy improved in the control group by 6 months, so that the difference in improvement between groups was not sustained.

We measured the effect of the mind-body program on psychological measures, mood, and quality of life. By 6 months, we found small changes that were significant but not clinically 
meaningful. At baseline, the sample was psychologically robust, with low scores for depressive symptoms that may explain the lack of change in both groups' scores over time. In addition, mindfulness scores indicated a high level of self-reported mindfulness at baseline. This level of mindfulness is likely the reason why we did not see a significant difference between groups at program completion, despite qualitative reports by participants of increased mindfulness after learning to meditate. The pilot work ${ }^{6}$ also found this change. Further refinement of mindfulness measures is needed to capture changes in mindfulness in the older population.

Our study was conducted in Western Pennsylvania and had a greater proportion of women, which may limit the generalizability of the findings. Intervention participants received information on a mind-body approach to pain reduction, whereas the comparison group did not receive education about pain, which may have biased responses to measures. Expectation of a positive treatment effect on pain was similar at baseline for both groups. Participation rates in the 8-week programs were high for each group (mean number of classes attended for each group was 6.6), but booster session attendance was low for both groups, which may have influenced responses over time. Our study also included 80 African American participants (28.4\%), which is unusual for mind-body studies that do not specifically target racial or ethnic minorities. The participation of a diverse sample may be a reflection of the interest in older adults in effective treatments for pain relief regardless of race or ethnicity.

\section{Conclusions}

We found that an 8-week mind-body program that taught mind-fulness meditation methods resulted in significant improvements in short-term physical function and long-term current and most severe pain intensity in the past week. The functional improvement was not sustained. Our clinical trial suggests that mindful-ness has a role in the treatment of chronic LBP in the older adult. Refinement of the mindfulness program could focus on enhancing the durability of functional improvement.

\section{Supplementary Material}

Refer to Web version on PubMed Central for supplementary material.

\section{Acknowledgments}

Funding/Support: This study was supported by grant R01 AG034078 from the National Institutes of Health.

\section{REFERENCES}

1. Institute of Medicine. Relieving Pain inAmerica: A Blueprintfor Transforming Prevention, Care, Education, and Research. Washington, DC: National Academies Press; 2011.

2. Patel KV, Guralnik JM, Dansie EJ, Turk DC Prevalence and impact of pain among older adults in the United States: findings from the 2011 National Health and Aging Trends Study. Pain. 2013; 154(12):2649-2657. [PubMed: 24287107]

3. Weiner DK, Kim YS, Bonino P, Wang T. Low back pain in older adults: are we utilizing healthcare resources wisely? Pain Med. 2006;7(2):143-150. [PubMed: 16634727] 
4. Clarke TCBL, Black LI, Stussman BJ, Barnes PM, Nahin RL Trends in the use ofcomplementary health approaches among adults: United States, 2002-2012. Natl Health Stat Report. 2015;(79):116.

5. Goyal M, Singh S, Sibinga EM, et al. Meditation programs for psychological stress and well-being: a systematic review and meta-analysis. JAMA Intern Med. 2014;174(3):357-368. [PubMed: 24395196]

6. Morone NE, Rollman BL, Moore CG, Li Q, Weiner DK. A mind-body program for older adults with chronic low back pain: results of a pilot study. Pain Med. 2009;10(8):1395-1407. [PubMed: 20021599]

7. Schmidt S, Grossman P, Schwarzer B, Jena S, Naumann J, Walach H. Treating fibromyalgia with mindfulness-based stress reduction: results from a 3-armed randomized controlled trial. Pain. 2011;152 (2):361-369. [PubMed: 21146930]

8. Morone NE, Greco CM, Weiner DK. Mindfulness meditation for the treatment of chronic low back pain in older adults: a randomized controlled pilot study. Pain. 2008;134(3):310-319. [PubMed: 17544212]

9. Morone NE, Greco CM, Rollman BL, et al. The design and methods of the Aging Successfully With Pain Study. Contemp Clin Trials. 2012;33(2): 417-425. [PubMed: 22115971]

10. Kabat-Zinn J Full Catastrophe Living: Using the Wisdom of YourBody and Mind to Face Stress, Pain, and Illness. New York, NY: Delacorte; 1990.

11. Newman AB, Bayles CM, Milas CN, et al. The10 Keys to Healthy Aging: findings from an innovative prevention program in the community. JAgingHealth. 2010;22(5):547-566.

12. Folstein MF, Folstein SE, McHugh PR. "Mini-Mental State": a practical method for grading the cognitive state of patients for the clinician. J PsychiatrRes. 1975;12(3):189-198.

13. Roland M, Morris R. A study of the natural history of back pain, I: development ofa reliable and sensitive measure of disability in low-back pain. Spine (Phila Pa 1976). 1983;8(2):141-144. [PubMed: 6222486]

14. YesavageJA, Brink TL, Rose TL, et al. Development and validation ofa geriatric depression screening scale: a preliminary report. J PsychiatrRes. 1982-1983;17(1):37-49.

15. Kovacs FM, Abraira V, Royuela A, et al. Minimal clinically important change for pain intensity and disability in patients with nonspecific low back pain. Spine (Phila Pa 1976). 2007;32(25): 2915-2920. [PubMed: 18246018]

16. Herr KA, Spratt K, Mobily PR, Richardson G. Pain intensity assessment in olderadults: use of experimental pain to compare psychometric properties and usability of selected pain scales with younger adults. ClinJPain. 2004;20(4):207-219.

17. Hays RD, Prince-Embury S, Chen H. RAND-36 Health Status Inventory. San Antonio, TX: Psychological Corp; 1998.

18. Magni G, Caldieron C, Rigatti-Luchini S, Merskey H. Chronic musculoskeletal pain and depressive symptoms in the general population: an analysis of the1st National Health and Nutrition Examination Survey data. Pain. 1990;43(3):299-30. [PubMed: 2293141]

19. Bandura A Recycling misconceptions of perceived self-efficacy. CognitTherRes. 1984;8(3): 231255.

20. Anderson KO, Dowds BN, Pelletz RE, Edwards WT, Peeters-Asdourian C. Development and initial validation ofa scale to measure self-efficacy beliefs in patients with chronic pain. Pain. 1995;63(1):77-84. [PubMed: 8577493]

21. Rosenstiel AK, Keefe FJ. The use of coping strategies in chronic low back pain patients: relationship to patient characteristics and current adjustment. Pain. 1983;17(1):33-44. [PubMed: 6226916]

22. Brown KW, Ryan RM. The benefits of being present: mindfulness and its role in psychological well-being. J PersSoc Psychol. 2003;84(4):822-848.

23. Linn BS, Linn MW, Gurel L. Cumulative Illness Rating Scale. JAm GeriatrSoc. 1968;16(5):622626.

24. Devilly GJ, Borkovec TD. Psychometric properties of the Credibility/Expectancy Questionnaire. J Behav TherExp Psychiatry. 2000; 31(2):73-86. 
25. Pocock SJ, Simon R. Sequential treatment assignment with balancing for prognostic factors in the controlled clinical trial. Biometrics. 1975;31(1): 103-115. [PubMed: 1100130]

26. O'Brien EM, Staud RM, Hassinger AD, et al. Patient-centered perspective on treatment outcomes in chronic pain. Pain Med. 2010;11(1):6-15. [PubMed: 19732374]

27. Towheed TE, Maxwell L, Judd MG, Catton M, Hochberg MC, Wells G. Acetaminophen for osteoarthritis. Cochrane Database Syst Rev. 2006; (1):CD004257. [PubMed: 16437479]

28. Hayden JA, van Tulder MW, Malmivaara AV, Koes BW. Meta-analysis: exercise therapy for nonspecific low back pain. Ann Intern Med. 2005; 142(9):765-775. [PubMed: 15867409]

29. Jackson T, Wang Y, Wang Y, Fan H. Self-efficacy and chronic pain outcomes: a meta-analytic review. J Pain. 2014;15(8):800-814. [PubMed: 24878675] 


\section{Key Points}

Question: Does a mind-body program improve function and reduce pain in older adults with chronic low back pain?

Findings: This randomized clinical trial of older adults compared a mindfulness meditation program and an education program. At program completion, function improved in the intervention group; current and most severe pain in the past week also improved significantly. Only current and most severe pain showed significant improvement at 6 months.

Meaning: A mind-body program may improve short-term function and provide some long-term pain relief. 


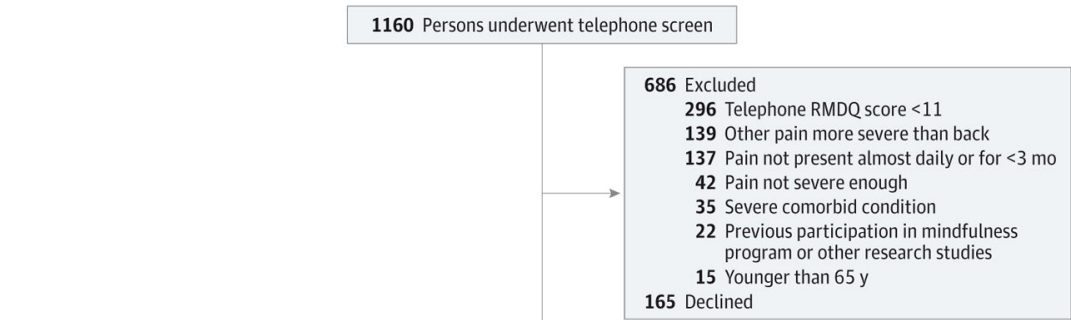

309 Signed informed consent

25 In-person screen ineligible 10 RMDQ scores $<11$

8 GDS scores $>20$

4 Physical examination

1 MMSE score $<24$

1 Younger than $65 y$

1 Previous participation in mindfulness

program

2 Declined after baseline and before

randomization

140 Randomized to intervention

136 Received allocated intervention

4 Did not receive allocated intervention

2 No longer interested

1 Serious illness

1 Unexpected family obligation

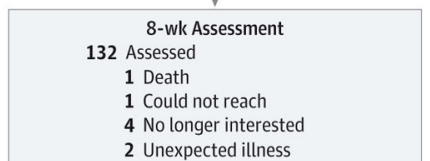

2 Unexpected illness

6-mo Assessment
118 Assessed
4 Missed assessment
10 Lost to follow-up
4 No longer interested
2 Unable to reach
1 Death
1 Termination by PI
1 Unexpected family obligation
1 Moved out of state
140 Included in primary analysis

142 Randomized to control

137 Received allocated interventio

5 Did not receive allocated intervention

2 No longer interested

1 Unexpected illness

1 Unexpected family illness

1 Unsatisfied with randomization

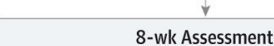

138 Assessed

1 Missed assessment

2 No longer interested

1 Unexpected illness 6-mo Assessment

135 Assessed

1 Missed assessment

3 Lost to follow-up

2 No longer interested

1 Unexpected ill

142 Included in primary analysis

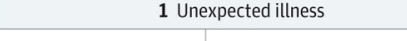

Figure 1. CONSORT Diagram of Study Participation

GDS indicates Geriatric Depression Scale; MMSE, Mini-Mental State Examination; PI, principal investigator; and RMDQ, Roland and Morris Disability Questionnaire. 


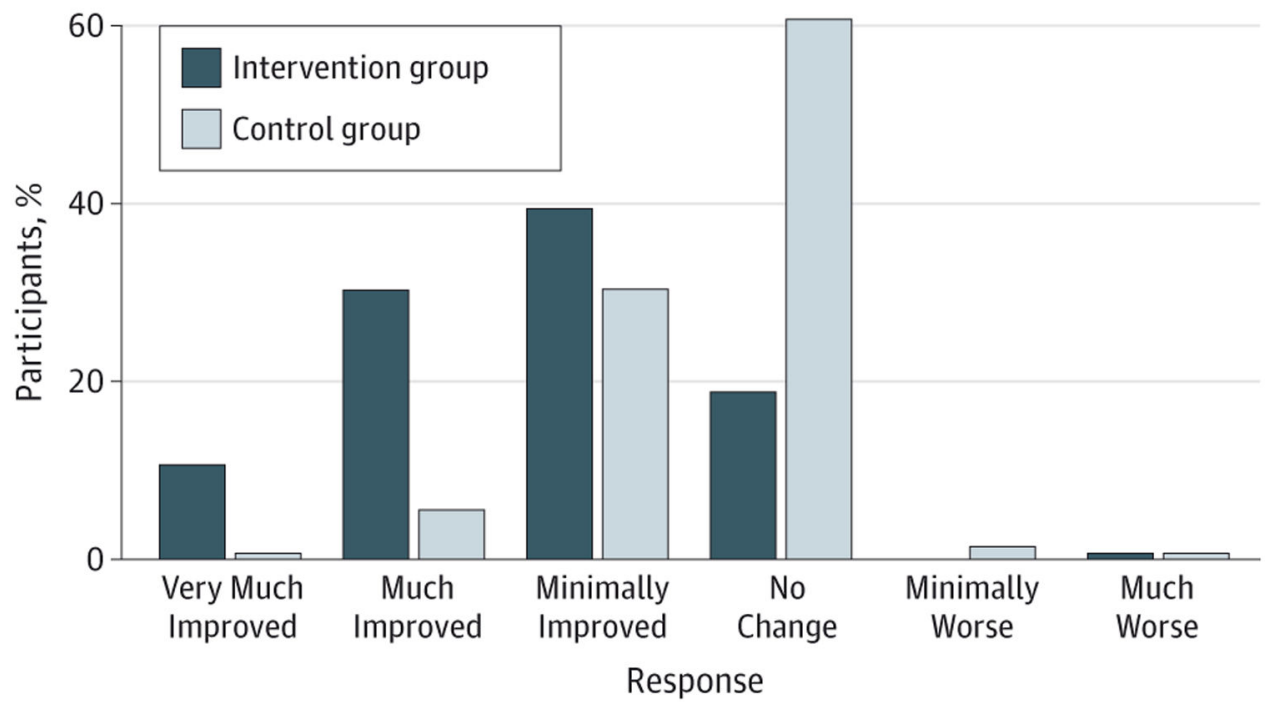

Figure 2. Global Impression of Change Findings

Participants in both groups answered the question "How much have your back symptoms changed as a result of the treatment provided in this study?" Assessment was performed at the end of each program ( 8 weeks). Improvement in back pain symptoms among the intervention participants was significantly greater than among control participants $(P<$. 001). 
골

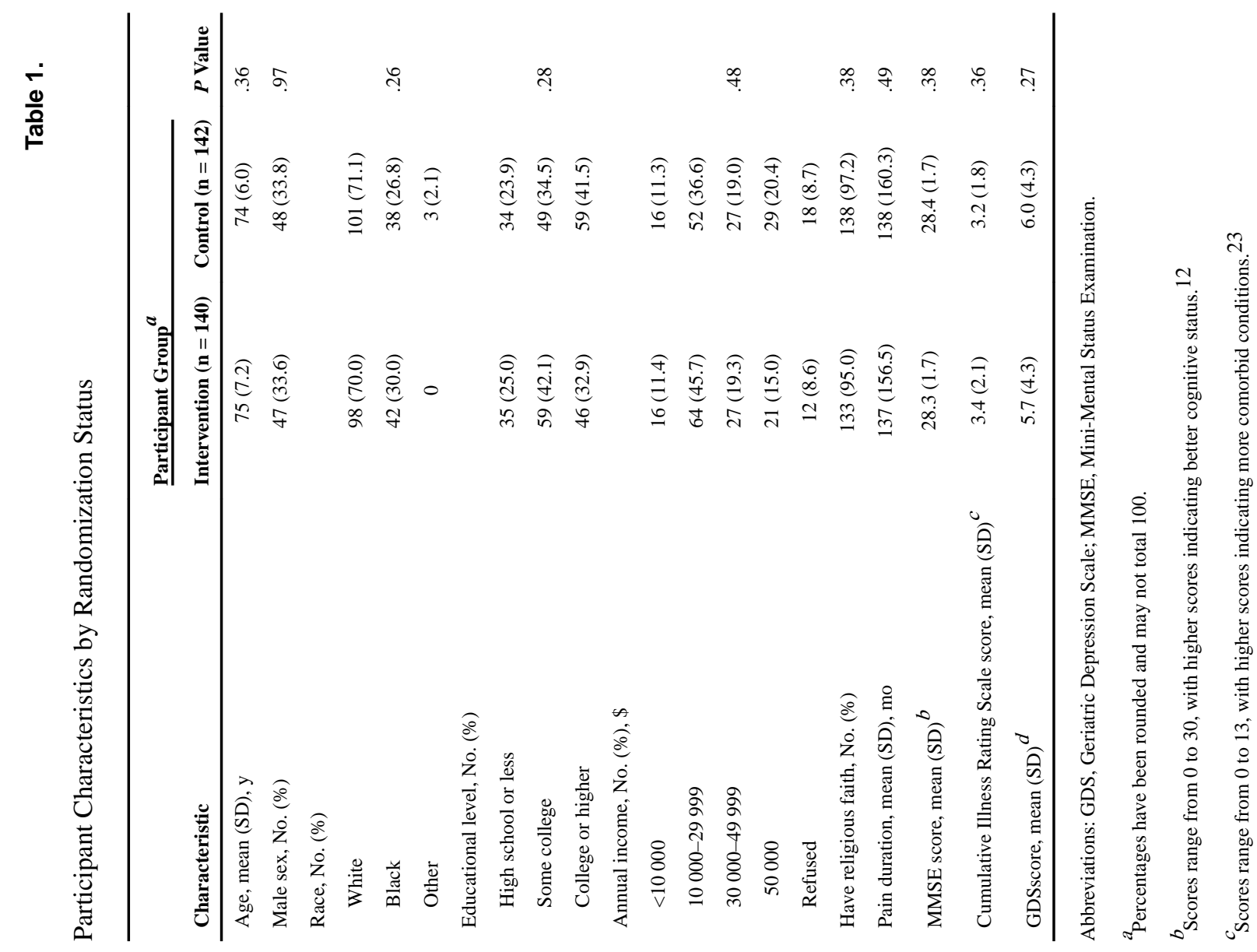




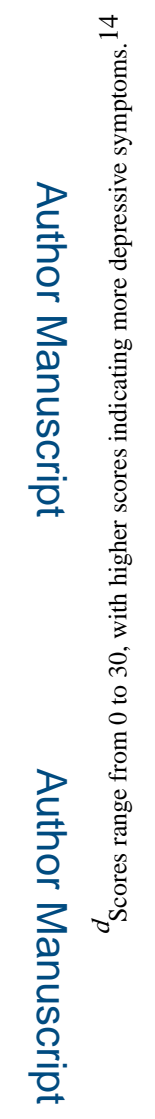

로을

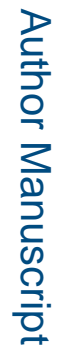

JAMA Intern Med. Author manuscript; available in PMC 2019 February 04. 


\section{을 \\ }

ב্

롤

ำ

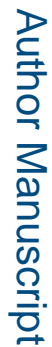

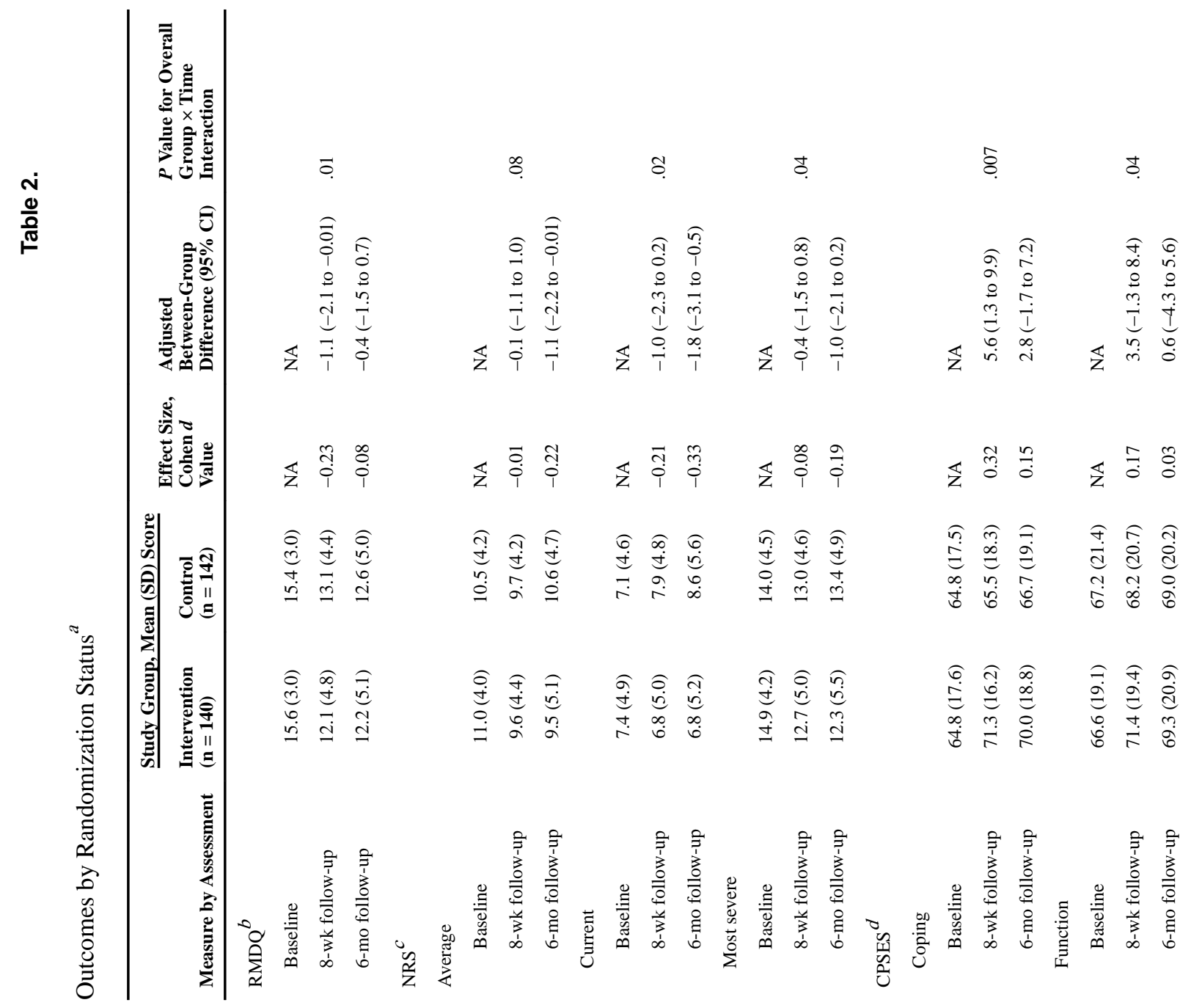

JAMA Intern Med. Author manuscript; available in PMC 2019 February 04 


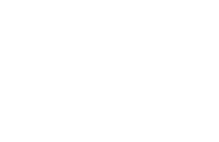

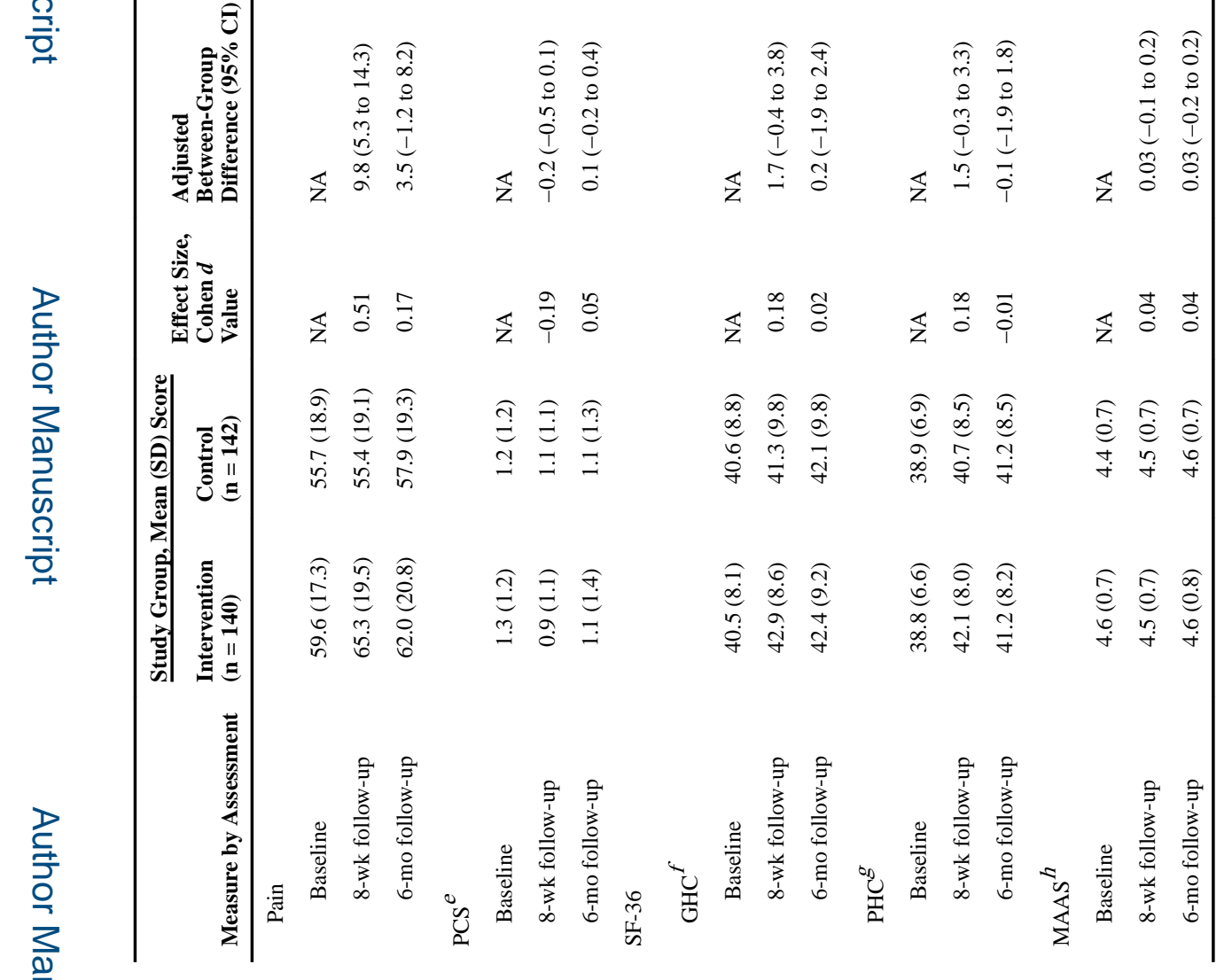
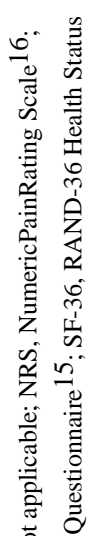

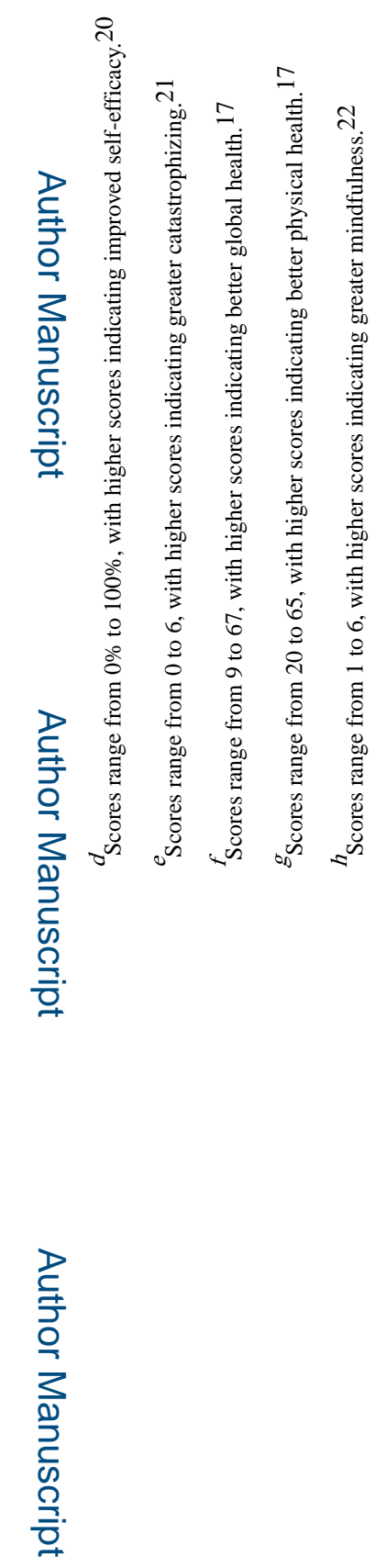

로을

JAMA Intern Med. Author manuscript; available in PMC 2019 February 04. 


\section{롤 \\ 올}

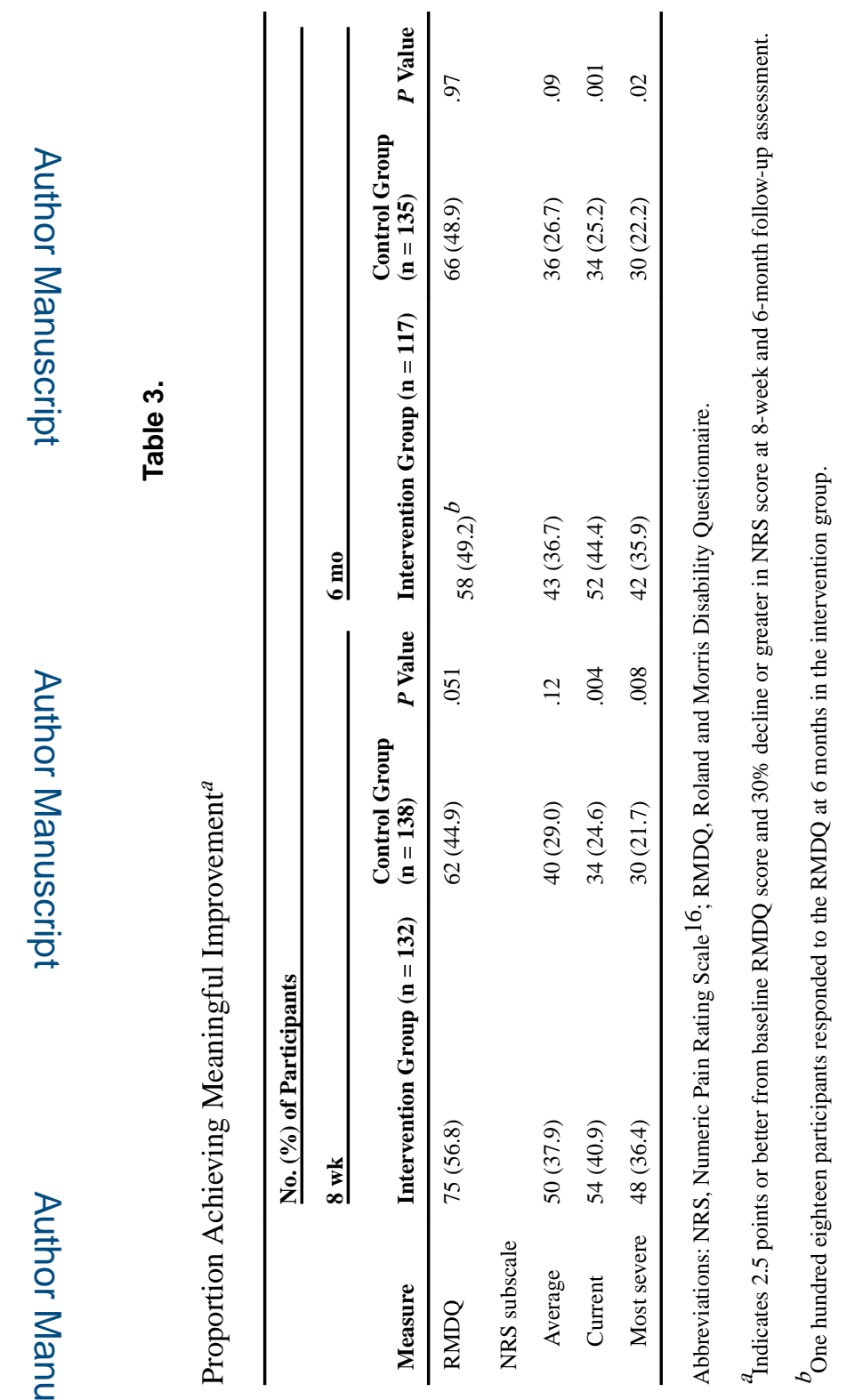

